\author{
Anđelka D. Pejović* \\ Universidad de Belgrado \\ Facultad de Filología \\ Luiza M. Valožić** \\ Universidad de Belgrado \\ Facultad de Filología
}

\title{
LA CONSTRUCCIÓN FRASEOLÓGICA [Vmov + prep + [los cerros de Úbeda]] Y SUS POSIBLES EQUIVALENTES CONTEXTUALES EN SERBIO
}

\author{
Original scientific paper \\ UDC 811.134.2'373.7=163.41 \\ https://doi.org/10.18485/kkonline.2021.12.12.12
}

En este trabajo presentamos la construcción fraseológica española [Vmov + prep + [los cerros de Úbeda]] y a partir de muestras de uso y función en contextos concretos identificamos sus matices de significado en español y ofrecemos sus posibles equivalentes prototípicos en serbio. También nos detenemos en las modificaciones de dicha construcción, igual que en las modificaciones de la construcción "base" [los cerros de Úbeda], en sus dos vertientes, la variación morfosintáctica y la funcional, para ver de qué modo afectan la traducción al serbio. A través del análisis de los significados de la construcción fraseológica española, sus modificaciones, la variación, y el análisis de cómo estos afectan la identificación de equivalentes en serbio, encontramos muestras concluyentes de la fijación cognitiva, que ha sido identificada como inherente a las construcciones fraseológicas.

Palabras clave: equivalencia, fraseología, corpus, traducción, gramática de construcciones, español, serbio.

\section{Introducción. Objeto de estudio, metodología y corpus}

La equivalencia en la diferencia es el problema central del lenguaje y la lingüística (Jakobson 1959: 233). El mismo concepto también es el eje de la traducción, que, según el mismo autor, supone «dos mensajes equivalentes en dos códigos diferentes» (Jakobson, 1959: 233). De ahí la importancia de los diccionarios bilingües para que ofrezcan una cuidadosa definición de carácter comparativo de todas las unidades correspondientes en su intensión y su extensión (Jakobson, 1959: 234).

En el ámbito de la fraseología, numerosos trabajos de investigación y práctica traductológica han mostrado, no obstante, que la equivalencia lexicográfica no es lo

\footnotetext{
*Facultad de Filología, Universidad de Belgrado, Studentski trg 3, 11000 Beograd; e-mail: andjelka.pejovic@fil.bg.ac.rs

**Facultad de Filología, Universidad de Belgrado, Studentski trg 3, 11000 Beograd; e-mail: luiza.valozic@fil.bg.ac.rs
} 
mismo que la equivalencia (con)textual (por ej. Torrent-Lenzen, 2009, 2012; Timofeeva 2012; Mellado Blanco, 2015; Sevilla Muñoz, 2015). En palabras de Mellado Blanco (2015: 155), «la equivalencia lexicográfica se diferencia de la equivalencia textual en que no pretende hacerse eco de toda la casuística posible de traducciones de un fraseologismo de la L1 en la L2». Por lo tanto, la definición de un fraseologismo en diccionarios monolingües o los equivalentes ofrecidos en diccionarios bilingües no siempre son la solución adecuada para una determinada traducción. A partir de los diccionarios fraseológicos del español Torrent-Lenzen (2009: 535-536) observa que:

«(a) muchas definiciones no son lo suficientemente precisas; (b) muchas definiciones han sido formuladas sobre la base de información insuficiente; (c) los diccionarios, en general, y el DFDEA en particular, se basan excesivamente en el significado recto a la hora de definir y a menudo renuncian ya de entrada a caracterizar y describir todos los elementos relevantes para la definición desde el punto de vista pragmático y sincrónico; (d) en relación con algunas locuciones falta registrar acepciones».

Ello se debe a la gran complejidad del significado fraseológico, gracias a lo que «las locuciones tienen la capacidad de adaptar su significado al contexto» (TorrentLenzen 2009: 544), y de esta forma «adquieren un único sentido» (Sevilla Muñoz, 2015: 96). También Timofeeva (2012: 420) advierte que «los valores que integran el significado de una UF ${ }^{1}$ se presentan como potenciales, y en el discurso manifiestan un desarrollo desigual en función de las circunstancias contextuales».

Teniendo en cuenta lo dicho, el objeto de nuestro estudio es la búsqueda de equivalencias contextuales de la construcción fraseológica española [Vmov + prep + [los cerros de úbeda]] en la lengua serbia, a partir de ejemplos reales de uso. Según los diccionarios consultados, la locución verbal (irse/salir) por los cerros de úbeda tiene un solo significado: 'apartarse mucho del asunto que se está tratando, esp. diciendo un sinsentido o algo que no viene al caso' (CLAVE). ${ }^{2}$ Partiendo de la definición lexicográfica, podría inferirse que el equivalente serbio, o uno de los equivalentes, sería la locución verbal obilaziti kao kiša oko Kragujevca. No obstante, el DLE registra la locución adverbial por los cerros de úbeda (con el significado 'por sitio o lugar muy remoto y fuera de camino. U. para dar a entender que lo que se dice es incongruente o fuera de propósito, o que alguien divaga o se extravía en el raciocinio o discurso').

\footnotetext{
${ }^{1}$ Unidad fraseológica

${ }^{2}$ Algunos diccionarios fraseológicos registran y definen esta locución de la siguiente forma: irse (o salir) por los cerros de Úbeda. $v(\mathrm{col})$ Divagar, o desviarse del tema. En lugar de IRSE o SALIR puede aparecer otro $v$ equivalente (DFDEA); echar/irse/salir(se) u.p. por los cerros de úbeda (inf.). Hablar fuera de propósito o disparatadamente (DFEM).
} 
Consecuentemente, el equivalente en forma de locución verbal mencionado no podría ser el adecuado, sino que habría que pensar en uno de categoría adverbial. Pejovic (2021), partiendo de los postulados de la gramática de construcciones, considera que en este caso es más apropiado observar dicha expresión como construcción, y a partir de los ejemplos extraídos del corpus indica que los cerros de úbeda es una construcción fraseológica léxicamente saturada, que a su vez suele formar parte de una construcción más amplia, que contiene una casilla vacía o slot en forma de verbo de movimiento seguido de la preposición correspondiente: [Vmov + prep + [los cerros de Úbeda]]. Observando el comportamiento de dicha construcción en el corpus, se ve claramente que en serbio le corresponde más de un equivalente, a pesar de que en los diccionarios monolingües de uso general pero también fraseológicos se registra un significado. De ahí que en este trabajo nos ocupemos de los posibles equivalentes contextuales de dicha construcción en serbio.

Dobrovol'skiï (2005: 361) subraya que «las diferencias semánticas entre fraseologismos no pueden ser predichas ni dentro de la misma lengua ni tampoco translingüísticamente», y que esas diferencias pueden descubrirse «solo mediante una investigación de su ámbito de uso». En este trabajo pretendemos aplicar precisamente esos postulados, y sobre la base de los rasgos semánticos, sintácticos y pragmáticos de dicha construcción, observados en el corpus, determinar sus equivalentes en serbio.

El corpus sobre el que se ha realizado el análisis consta de 68 coocurrencias, extraídas de los corpus CREA y CORPES XXI por Pejovic (2021). Asimismo, partimos de los matices de significado de dicha construcción señalados por la autora. Nuestro objetivo consiste en sistematizar las equivalencias contextuales correspondientes, según los matices de significado identificados, lo que podría ser útil a la hora de redactar un diccionario (fraseológico) bilingüe español-serbio.

Antes vamos a detenernos brevemente en el problema de la equivalencia, en el ámbito de la fraseología en particular.

\section{El problema de la equivalencia interlingüística}

En el ámbito de la traductología existen numerosos trabajos sobre la equivalencia y diversos tipos de equivalentes. ${ }^{3}$ Schellheimer (2016: 95) recuerda, citando a Bolaños Cuellar (2002), que en la traductología se reconocen dos tipos de enfoque, mutuamente excluyentes: «los enfoques orientados a aspectos lingüísticos y textuales

3 Véanse, por ejemplo, Snell-Hornby (1986), Newmark (1988), Eismannn (1989), Korhonen (1991), Baker (1992), etc. 
de la traducción, y los enfoques orientados a aspectos extratextuales». ${ }^{4}$ Los primeros se remontan a los comienzos de los estudios de traducción, en los que la investigación sobre la relación entre el texto original y el texto meta tenía un carácter claramente estructuralista. Es decir, «la traducción se consideró como una operación meramente lingüística, en la que el traductor descifra un código en lengua de origen y lo transcodifica en la lengua meta» (Schellheimer, 2016: 96); por consiguiente, se trata de una equivalencia sistémica. La introducción del aspecto textual, además del lingüístico, se debe a Catford, quien habla de la equivalencia formal y la textual (Schellheimer, 2016: 98). Nida y Taber, por su parte, ven la traducción como «un acto comunicativo», y para ellos, «traducir consiste en reproducir un mensaje del texto original mediante el equivalente más natural posible en la lengua meta» (Schellheimer, 2016: 99). Con el tiempo, y con el desarrollo de la pragmática, los especialistas empiezan a percatarse de la importancia del uso, del habla, y conciben la traducción como «un fenómeno condicionado por la situación comunicativa y la cultura en las que se desarrolla» (Schellheimer, 2016: 107). Asimismo, se va desplazando el foco del texto original hacia el texto meta, y se refuerza el papel y la importancia del traductor. La noción de equivalencia se redefine, y se ve como un concepto dinámico y no estático (Schellheimer, 2016: 116). Lo mismo es válido para la traducción de las unidades fraseológicas.

\subsection{La equivalencia en el ámbito de la traducción de las unidades fraseológicas}

Corpas Pastor $(2001,2003 a)$ señala tres principales pasos en la traducción de las unidades fraseológicas: el reconocimiento o la identificación de la UF en el texto origen, su correcta interpretación, y, finalmente, la transmisión de su significado y sus valores pragmático-textuales al texto meta. Este tercer paso, entonces, consiste en «buscar en el "baúl" fraseológico aquellas unidades que presenten un grado más alto de equivalencia con respecto a la UFO», que se ve obstaculizado por el «anisomorfismo fraseológico de las lenguas» (Corpas Pastor 2001: 68). Debido a esa inequivalencia de valores semántico-pragmáticos, una UF de la lengua de origen no siempre tiene como equivalente una UF en la lengua meta, sino que se puede transmitir mediante una unidad léxica simple, un calco (Corpas Pastor 2001: 71), o a través de una descripción; incluso puede omitirse (si se considera no necesaria o presenta demasiadas

\footnotetext{
${ }^{4}$ En este trabajo, no obstante, no pretendemos profundizar en los enfoques y las corrientes en la traductología; para ello remitimos al excelente estudio realizado por Schellheimer (2016).
} 
dificultades de traducción), pero también puede compensarse en algún otro segmento del texto. En resumen, la equivalencia puede ser absoluta o total, parcial y nula.

Varios estudios posteriores indican el carácter relativo de estos niveles de equivalencia y muestran que los equivalentes supuestamente existentes en dos lenguas en realidad no lo son, o no lo son en todos los contextos. Tal vez por eso señale Dobrovol'skiï (2005: 363) que «desde el punto de vista traductológico, parece más importante conocer todas las propiedades sintácticas, semánticas y pragmáticas de un fraseologismo dado en L1 que tener una lista de todos los posibles equivalentes en L2, porque la adecuación del equivalente depende en cada ocasión de condiciones contextuales que no pueden ser anticipadas.»

Torrent-Lenzen (2012), por su parte, también cuestiona dichos tipos de equivalencia, obtenidos a partir de los estudios contrastivos, porque no suelen tomar en consideración el contexto. La autora distingue las siguientes tres fases en el proceso de traducción: «a) reconocer la unidad fraseológica como tal (...); b) averiguar o analizar su contenido semántico-pragmático en el contexto dado y las funciones que esa unidad fraseológica desempeña en el texto (modificaciones, actualización de diferentes planos del significado, etc.) (...); y c) encontrar una correspondencia en la lengua terminal». Recuerda que «en la segunda fase radica un momento esencial en todo proceso de traducción, ya que uno de los objetivos básicos de la traducción es la búsqueda de la equivalencia comunicativa (cf. Lawick 2009: 18-21)». De ahí que sea muy importante entender el texto original, y de ahí la importancia del significado de las unidades fraseológicas, como subraya la autora. Para ello, el contexto mismo juega un papel crucial e influye en la técnica de traducción de una determinada locución, puesto que «las locuciones son construcciones esencialmente pragmáticas» (TorrentLenzen, 2012: 281). La autora, por tanto, opera con el término equivalente (fraseológico) contextual, que «supone una equivalencia fraseológica o no fraseológica solo válida en un contexto determinado» (Torrent-Lenzen, 2012: 281). De ello se deriva que un mismo fraseologismo en $L 1$ puede tener más de un equivalente en $L 2$, dependiente del contexto, y no necesariamente en forma de unidad fraseológica, lo cual se muestra también en nuestro caso, como veremos a lo largo del trabajo. Lo importante es que el equivalente elegido cumpla la misma función comunicativa que la unidad fraseológica del texto original. La autora considera, por tanto, que puede hablarse de los conceptos de equivalencia plena, parcial y nula solo en casos de reproducción fraseológica. 
Timofeeva (2012) también apunta hacia la importancia del nivel discursivo, y considera que, a pesar de las ventajas y los logros del enfoque funcional, es necesario adoptar uno nuevo, que «ha de partir de la reformulación de la significación fraseológica, cuyo estudio (...) se ha de articular en dos niveles, el de significación convencionalizada y el de discurso» (Timofeeva 2012: 429). Entonces, en la práctica traductológica en el ámbito de la fraseología se hace cada vez más patente la importancia del significado fraseológico, cuya comprensión depende de su uso en discursos concretos.

Una buena muestra de la debilidad o la no estabilidad de la tipología de los equivalentes obtenidos a partir de los estudios contrastivos son los casos de los fraseologismos que se consideran universales, que supuestamente presentan equivalencia absoluta en varias lenguas, europeas principalmente; esas expresiones se conocen como europeísmos (Morvay, 1996; Corpas Pastor, 2001) o internacionalismos fraseológicos (Zholobova, 2005). ${ }^{5}$ A este respecto cabe destacar que unos análisis más detallados y más recientes han demostrado que no siempre se trata de equivalentes absolutos o plenos (por ej. Dobrovol'skiï, 2005), sino que más bien se trata de pseudo-equivalentes (Szyndler, 2014) o cuasi-equivalentes (Szerszunowicz, 2016), puesto que «estos "europeísmos" en las correspondientes lenguas viven su vida propia, o sea, funcionan integrados en el sistema lingüístico y fraseológico del idioma dado» (Morvay, 1996: 719). Según Dobrovol'skiï (2005: 360), «la existencia de paralelismos en el "significado nuclear" no implica necesariamente una equivalencia perfecta en el uso lingüístico». La inequivalencia absoluta se debe a las diferencias en cuanto a la función que cumplen en el texto, las restricciones de uso, el número de los significados desarrollados, el carácter diafásico, la fuerza ilocutiva, el potencial derivacional etc., por lo que a menudo se comportan como falsos amigos. ${ }^{6}$ O, en palabras de Corpas Pastor (2003b: 254), «los supuestos casos de UFS totalmente equivalentes tienden a convertirse en equivalentes parciales, una vez examinadas sus condiciones de uso, frecuencia, contenido semántico, connotaciones e implicaturas, etc.». Es decir, las diferencias, aunque muy sutiles, se dan en los tres niveles señalados por Dobrovol'skiï (2005): sintáctico, semántico y pragmático.

Mellado Blanco (2015) diferencia la equivalencia en el nivel sistémico, lexicográfico y textual, indicando que:

\footnotetext{
${ }^{5}$ Para algunos ejemplos en serbio, véase Trivic 2013, quien analiza las locuciones con el componente ojo que presentan equivalencia absoluta en el plano formal, semántico y funcional.

${ }^{6}$ Para ejemplos de falsos amigos fraseológicos del español y el serbio, véase Pejovic 2013.
} 
«la equivalencia sistémica es independiente de las coordenadas deícticas de tiempo y espacio, a diferencia de lo que sucede en el plano textual (...); la equivalencia textual es unívoca y está siempre vinculada a un texto, perdiendo fuera de este su validez absoluta (...); la equivalencia funcional, propia del nivel lexicográfico y textual (...) debe obtenerse mediante el análisis del comportamiento prototípico de las unidades fraseológicas en el nivel del discurso, para lo cual es esencial saber discriminar los usos típicos de los periféricos o poco representativos (Mellado Blanco 2015: 154, 155).

La autora identifica seis parámetros de la equivalencia interlingüística en el nivel sistémico, además del significado denotativo que han de compartir los equivalentes. Dichos parámetros son los siguientes: (1) «componentes léxicos que integran el fraseologismo», (2) «estructura morfosintáctica y función sintáctica», (3) «imagen en la que se apoya el significado literal», (4) «extensión y estructura semántica», (5) «componente connotativo-pragmático, referido a posibles marcas diatópicas, diastráticas y diafásicas, registro, connotaciones, situaciones de uso, modificaciones, implicaturas del hablante, frecuencia de uso, etc.», y (6) «valencia sintáctica y semántica (combinatoria sintagmática)» (Mellado Blanco, 2015: 160-169). Como indica, los tres últimos son los más relevantes en el plano de la equivalencia lexicográfica.

Sevilla Muñoz (2015: 96) sostiene que los factores de equivalencia del nivel léxico pueden dar como resultado correspondencias válidas en el nivel textual en numerosas ocasiones, pero esto no siempre es así, «lo que obliga a buscar otra correspondencia acorde con la naturaleza del texto». En este sentido, entiende la traducción fraseológica y paremiológica «como la traducción de textos con presencia de UF (sintagmas fraseológicos, enunciados fraseológicos, esquemas sintácticos y paremias), de modo que la prioridad en la traducción fraseológica y paremiológica es el mantenimiento de las características del TO en el texto meta (TM) y no la traducción de cada una de las UF por otra UF similar en la lengua de llegada (...)». Debido a ello, y en línea con las conclusiones de otros autores que hemos visto, la traducción fraseológica y paremiológica se abre a diferentes posibilidades, y «así la traducción de una UF podrá ser una UF del mismo tipo, una UF de otra categoría, una combinación libre de palabras o una palabra» (Sevilla Muñoz, 2015: 97). 


\section{La construcción fraseológica [Vmov + prep + [los cerros de Úbeda]]: forma, significado y posibles equivalentes contextuales en serbio}

Como hemos dicho, consideramos que la locución propiamente dicha es la construcción los cerros de úbeda, que, desde el punto de vista construccionista, está léxicamente saturada. A partir de los ejemplos del corpus (10), puede decirse que su significado es 'lugar remoto, apartado, desconocido':

Los socialistas afirman que los portavoces de los tres grupos que conforman esta oposición están en campaña electoral y se constituyeron en comisión de investigación "en un xantar celebrado en Tomiño", y que "en cualquier otro opíparo xantar en los cerros de Úbeda se constituirán en Tribunal Superior de Justicia de Nigrán". (2002; «REDACCIÓN - Nigrán». Faro de Vigo; España; CORPES XXI)

(...) mismo fray Juan lo repetiría en sus momentos decisivos: "Buscando mis amores" subiré al Carmelo, bajaré a la Cartuja, llegaré a Duruelo, pasaré por Pastrana; "Buscando mis amores" me descolgaré por los muros toledanos, me escaparé al Calvario, entraré en Granada, y me perderé por los cerros de Úbeda... (2003; «ISMAEL BENGOECHEA, OCD». San Juan de la Cruz; España; CORPES XXI)

La otra película, me atreveré a decirlo, es muy buena, a pesar del personaje central de la misma, el legendario Indiana Jones. Con ser la película una especie de "pandemónium" de los buenos tiempos de Bond, los nazis y unas posibilidades de infarto, la acción no decae y el héroe acaba yéndose por los cerros de Úbeda. Una síntesis de la demencial situación humana actual, pero en sueño, al "ralentí". (1989; Prensa; Cine y Vídeo; ABC, 23/08/1989; España; CREA)

Sus equivalentes en serbio podrían ser los siguientes: bespuće, nedođija (col), nigdina (col), kraj sveta (col). Aunque los diccionarios fraseológicos del español registran la locución (adverbial o verbal) como coloquial, consideramos, a base de los ejemplos de nuestro corpus, que puede pertenecer tanto al registro informal como formal. Los equivalentes serbios, excepto el primero, sin embargo, connotan más que la construcción española.

La construcción en cuestión suele combinarse con determinados verbos de movimiento (seguidos de preposiciones), formando de este modo una construcción fraseológica más compleja: [Vmov + prep + [los cerros de Úbeda]]. El análisis de los ejemplos extraídos de los corpus CREA y CORPES XXI parece mostrar que esta construcción tiene más de un significado, o que contiene matices de un mismo significado más amplio, ya que las fronteras entre unos y otros a veces no son muy nítidas. A nuestro juicio, estos son los siguientes: (1) 'alejarse en los pensamientos; 
estar despistado, distraído; no enterarse de algo', (2) 'desviarse del asunto del que se trata en el discurso, alejarse del tema', (3) 'desviarse del asunto del que se trata en el discurso, evitando el tema', (4) 'hablar o pensar demasiado, hasta de lo que no viene a cuento', (5) 'inesperadamente, decir algo incongruente, fuera de propósito, algo que no tiene que ver con el tema del discurso'. El más frecuente, según nuestro análisis, es el tercero (18 ejemplos), seguido del segundo (16 ejemplos, de los que 10 presentan algún tipo de modificación) y el cuarto (12 ejemplos).

En cuanto a los equivalentes contextuales en serbio, vamos a presentarlos siguiendo el modelo lexicográfico de Penadés Martínez (2021), adaptado en nuestro caso, porque consideramos que permite el desglose del significado de la construcción original en español.

\section{PALABRA CLAVE: cerro}

LEMA, SIGNIFICADO y EQUIVALENTES:

[los cerros de Úbeda] constr fras n 'lugar remoto, apartado, desconocido' bespuće $f$, nedođija $f(\mathrm{col})$; nigdina $f(\mathrm{col})$; kraj sveta $S N(\mathrm{col})$

EJEMPLOS:

- (...) solidaridad de desterrados a los cerros de Úbeda por la dictadura del fulgor ético que sanciona a no contrastar objeto y voz y que obliga a modular un equivalente del nocturno andrajo impresentable (1979; Manuel Longares, La novela del corsé; Novela; España; CREA)

- Los socialistas afirman que los portavoces de los tres grupos que conforman esta oposición están en campaña electoral y se constituyeron en comisión de investigación "en un xantar celebrado en Tomiño", y que "en cualquier otro opíparo xantar en los cerros de Úbeda se constituirán en Tribunal Superior de Justicia de Nigrán". (2002; «REDACCIÓN - Nigrán». Faro de Vigo; España; CORPES XXI)

REGISTRO: informal o neutro

LEMA (construcción compleja), SIGNIFICADO Y EQUIVALENTES:

[V + prep + [los cerros de Úbeda]] constr fras $v$ (estar despistado, distraído; alejarse en los pensamientos) biti $v$ (negde) u (nekom) svom svetu; otići / odlaziti $v$ u (neki) svoj svet

VARIACIÓN DE CASILLA Y EJEMPLOS: 


\section{$[V+$ prep+ [los cerros de Úbeda $]]$ \\ estar \\ andar \\ irse}

- Seguro que tú le pareces un irresponsable, un mentiroso y un maniático que lo mismo lo piensa todo cien veces o que está por los cerros de Úbeda soñando con las musarañas. ¿No es verdad? (2003; Fernando Iwasaki, «La jumelle fatale». Un milagro informal; Perú; CORPES XXI)

- Juan, que siempre parece que anda distraído, como por las nubes, los cerros de Úbeda o de Anacapri, demostró tener un enorme sentido práctico. (1977; Jorge Semprún, Autobiografía de Federico Sánchez; Novela; España; CREA)

- Cuando abre la mano se va, según quedó patente en la antológica del Reina Sofía, por los cerros de úbeda, o incluso más allá. Pero bueno, da lo mismo. Destreza aparte, Balthus es un evocador. Esto es, lo suyo consiste en trasladar al lienzo presencias humanas que nos impresionan como algo que se ve o entrevé a través del ojo de una cerradura. (...) (1996; ABC Cultural, 03/05/1996; Arte y cultura en general; España; CREA)

REGISTRO: informal o neutro

LEMA (construcción compleja) SIGNIFICADO Y EQUIVALENTES:

[V + prep + [los cerros de Úbeda]] constr fras $v$ 1. (alejarse de un tema) skrenuti s teme, udaljiti se od teme $v$ 2. (evitar un tema) obilaziti kao kiša oko Kragujevca constr $v(\mathrm{col})$; okolišati $v$ 3. (hablar o pensar demasiado) rasplinuti se $v$ VARIACIÓN DE CASILLA Y EJEMPLOS:

[V + prep + [los cerros de Úbeda] $]$

irse

ir

salir

$\cdots$

- Permíteme que me vaya por los cerros de Úbeda y, antes de centrarte la jugada de un nuevo restorán, te referencie un par de buenas lecturas que tienen bastante que ver con Asturias y Koldo Miranda (...) (2010; David de Jorge E., Con la cocina no se juega; España; CORPES XXI) 
- "Pero bueno, doctora, se está volviendo a ir usted por los cerros de Úbeda y no me cuenta lo principal, qué tal le iban las cosas con ese chico de la voz bonita." (1992; Carmen Martín Gaite, Nubosidad variable; Novela; España; CREA)

- iQue mucho habláis y andáis por los cerros de Úbeda, pero aún no habéis acabado de decirnos, a la señora ama y a mí, quién es ese huésped amigo del otro marido de doña Juana! (1995; María Luz Melcón, Catalina de Cervantes I. Boda en Esquivias; Teatro; España; CREA)

REGISTRO: informal o neutro

LEMA (construcción compleja) Y SIGNIFICADO:

$[\mathbf{V}+$ prep $+[$ los cerros de Úbeda] $]$ constr fras $v \mathbf{1}$. (decir algo inesperadamente y fuera de propósito) lupiti / lupati / lupetati $v$ (col)

VARIACIÓN DE CASILLA Y EJEMPLOS:

[V + prep + [los cerros de Úbeda] $]$

salir

salirse

$\cdots$

- Y aunque hubiera deseado decirle qué manía tienes hija, me callé la boca, preocupada porque fuera a dar lugar a faltas de respeto entre las dos, que yo cuidaba mucho. Entonces le dio uno de sus arrebatos y me salió por los cerros de Úbeda, hablándome de las torres de la catedral de la Almudena de Madrid. (2002; María Paz Díaz, Amor en Florencia; España; CORPES XXI)

- He traído unos zapatos espantosos - salió Helen por los cerros de Úbeda. (2002; Antonio Gala, «REUNIÓN DE TRABAJO EN PRIMAVERA». Los invitados al jardín; España; CORPES XXI)

REGISTRO: informal o neutro

Como se observa en los ejemplos, la fijación cognitiva (Mellado Blanco 2020) presente en la construcción [los cerros de Úbeda] es la que permite que dicha expresión se combine con determinados verbos y preposiciones sin que se pierda la imagen "nuclear", la imagen de fondo de la locución. Mellado Blanco explica que «cuanto mayor es la fijación cognitiva (entrenchment), mayor tendencia a la variación, a la productividad y a la esquematicidad» (Mellado Blanco, 2020: 19). 
La locución los cerros de Úbeda y su forma compleja [V + prep + [los cerros de Úbeda]] no presenta una gran frecuencia en el corpus (68 ocurrencias), pero aun así muestran la capacidad de adoptar «distintas formas léxicas, (...) relacionadas entre sí de manera asociativa y creadas en virtud de un proceso de analogía» (Mellado Blanco, 2020: 30), que se debe precisamente a la fijación cognitiva, que como tal hace que el significado de la expresión sea reconocido aunque esta tenga una forma diferente. Encontramos 15 ejemplos donde las construcciones de partida (la simple y la compleja) presentan algún tipo de modificación. Dichas modificaciones son principalmente morfosintácticas, y se reflejan en la sustitución del elemento Úbeda por otro (por ejemplo, Uganda, Tucumán, Lenny Kravitz, revolución), la ampliación de la construcción base mediante un adjetivo (por ejemplo, los cerros de úbeda políticos, unos imposibles cerros de úbeda), o en el cambio de la función sintáctica (voz de andar por los cerros de úbeda) etc. Los más frecuentes son los casos de sustitución del elemento úbeda por otro, en la construcción empleada con el significado 'desviarse del asunto del que se trata en el discurso, alejarse del tema'; por ejemplo:

GERMINAL: La verdad es que me desconcierta este último giro de nuestro coloquio. Yo comenzaba a hablarles de Sinapia y ustedes se me han ido por los cerros de la revolución. Pero lo cierto es que no hay país más estable ni menos propenso a las convulsiones subversivas que éste del que les hablo. (1983; Fernando Savater, Vente a Sinapia. Una reflexión española sobre la utopía; Teatro; España; CREA)

-Vaya, yo creía que todos los negros éramos iguales - comentó. Al menos, en mi poblado es así. Claro que allí no andamos todos vestidos de chófer. (...) Pero no nos vayamos por los cerros de Uganda. He venido a traerle un mensaje. (2001; Eduardo Mendoza, La aventura del tocador de señoras; España; CORPES XXI)

Con Maragall hay más simetría, pero me diferencia que yo tiendo menos a una poética difusa de la política. Él sabe divagar por los cerros de la política e incluso ir más allá. (1995; Prensa; Política; La Vanguardia, 16/11/1995; España; CREA)

Ya en la mesa, relajados y con las copas llenas y los platos rebosantes, Tanis le lanzó un cargamento de preguntas al que Yupanqui contestó como le vino en gana, es decir, yéndose literalmente por los cerros de Tucumán, el lugar a donde fue a parar con menos de diez años, donde cantan las zumbas más lindas. (2014; Nativel Preciado, Canta solo para mí; España; CORPES XXI)

En el curso de un directo poderoso como pocos, el quinteto anduvo muy atinado al mezclar en su punto justo reggae y blues, en lo que fue tal vez el mejor hallazgo del lote. Las incursiones de Walker y los Bosstalkers por los cerros de Lenny Kravitz, 
en una suerte de rock-soul superficial y edulcorado, resultaron por contraposición lo más flojo de la noche. (1995; Prensa; Música; La Vanguardia, 16/11/1995; España; CREA)

En los ejemplos citados, el elemento nuclear es [los cerros de Úbeda], donde 'los cerros' está tan asentado en la fijación cognitiva que permite que la posición de "Úbeda" se rellene con otro elemento. Esto es posible porque la variabilidad es «una característica inherente y propia de las unidades fraseológicas» (Corpas Pastor, 2001: 74). Debido a ello, «las UF no son estáticas, sino que se encuentran en interconexión con otras construcciones por medio de relaciones horizontales, con las cuales pueden surgir interferencias o lapsus, que no necesariamente tienen por qué ser percibidas por el hablante como desviaciones de una 'norma' y no interfieren el proceso comunicativo» (Mellado Blanco, 2020: 31).

Por las mismas razones también son posibles las "modificaciones" pragmáticas, como la que ilustra el siguiente ejemplo de nuestro corpus, donde la construcción de partida es empleada con un valor irónico:

«Reitero mi felicitación al autor de la nota y comprendo perfectamente que haya necesitado cuarenta días (desde el 18 de marzo al 28 de abril) para redactarla. En menos tiempo habría resultado imposible dar un rodeo por tan frondosos jardines retóricos: la pluralidad y diversidad de la Junta, la imparcialidad de su presidente, la independencia de todos los miembros, su decisión de mantenerse al margen de enfrentamientos políticos y empresariales, el fomento de la solidaridad entre los periodistas, su alejamiento de litigios personales y su exhortación a las partes al respeto mutuo y de la libertad de expresión de "contrario", para llegar, al final de tanto rodeo, a los cerros de Úbeda o al territorio de Babia. (...).» (1997; ABC Electrónico, 29/04/1997; Medios de comunicación; España; CREA)

Las modificaciones de las construcciones españolas influyen o pueden influir en su traducción al serbio, y la modificación de la unidad fraseológica en la lengua de partida también puede condicionar la presencia de las modificaciones en la unidad de la lengua meta, sin que ello impida la descodificación del significado. Esto reafirma una vez más la «fijación cognitiva» de las UF en ambas lenguas. Así, por ejemplo, en el caso de

Ya en la mesa, relajados y con las copas llenas y los platos rebosantes, Tanis le lanzó un cargamento de preguntas al que Yupanqui contestó como le vino en gana, es decir, yéndose literalmente por los cerros de Tucumán, el lugar a donde fue a parar con

menos

de

diez años, 
donde cantan las zambas más lindas. Pero nació en la provincia de Buenos Aires, más cerca de Rosario que de la capital (...) (2014; Nativel Preciado, Canta solo para mí; España; CORPES XXI), la construcción modificada podría traducirse como obilazeći kao kiša oko Tukumana, lo que sería una modificación de la locución obilaziti kao kiša oko Kragujevca.

Aunque la mayoría de los ejemplos con la construcción modificada tienen el significado 'alejarse de un tema', la traducción de cada uno es particular. Así, en un contexto la mejor opción es la creación de un neologismo en la lengua meta (por ejemplo, okolišac, para un buen cerro de úbeda). En otro contexto, a la construcción fraseológica de origen le corresponde una locución en la lengua meta que no puede aplicarse a otros casos; la construcción española no se refiere a un lugar remoto sino, por extensión metafórica, a un tiempo remoto en cuanto al momento presente, lo que en serbio podría expresarse con la locución od Kulina bana (...vraćanje na početak od Kulina bana...):

¿Podremos afrontar definitivamente la obra de Julio Romero de Torres, a una buena distancia ya de la transición democrática de nuestro país? Lo que dicha transición comportó necesariamente de secularización cultural y, al menos así hay que esperarlo, de posibilidad de llevar a cabo una reflexión crítica sobre nuestro pasado, implica una reflexión sobre nuestra identidad fantasmal capaz de discernir entre eso que verdaderamente somos y las múltiples pesadillas que han acompañado nuestra forma histórica de ser. No creo que sea remontarse a los cerros de Úbeda el plantear, en los términos expuestos, la interrogación anterior (...) (1998; Calvo Serraller, Francisco, Paisajes de luz y muerte. La pintura española del 98; Pintura, España; CREA)

En resumen, la equivalencia contextual puede adoptar diferentes formas; así, a una unidad fraseológica en L1 le puede corresponder en L2 una UF en su forma original o una UF modificada, pero también una descripción, una unidad léxica simple e incluso un neologismo.

\section{Conclusiones}

En el presente trabajo se han expuesto algunas reflexiones sobre la forma, el significado y el comportamiento contextual de la construcción fraseológica española cuyo elemento nuclear es [los cerros de Úbeda]. Consideramos que los cerros de Úbeda es una locución o una construcción fraseológica nominal, léxicamente saturada. Sus equivalentes en serbio pueden ser bespuće, nedođija (col), nigdina (col), kraj 
sveta (col). Las modificaciones de dicha locución (la sustitución del elemento Úbeda por otro, sea un topónimo o un sustantivo común) confirman que dicha construcción está fijada en la cognición de los hablantes de español, y es precisamente la fijación cognitiva la que permite que el significado de la construcción sea reconocido, aunque esta se materialice en una forma diferente. También sostenemos que dicha locución forma una construcción compleja al combinarse, debido a su valor semántico ('lugar remoto, apartado, desconocido'), con determinados verbos de movimiento seguidos de preposición; presentamos esa construcción mediante el esquema [ $\mathrm{V}$ mov + prep + [los cerros de Úbeda]].

A partir de las 68 ocurrencias extraídas de los corpus CREA y CORPES XXI, hemos identificado 5 significados de dicha construcción compleja y hemos constatado que les corresponden los siguientes equivalentes en serbio:

[estar/andar/irse + prep + [los cerros de Úbeda]] (estar despistado, distraído; alejarse en los pensamientos) biti $v$ (negde) u (nekom) svom svetu; otići / odlaziti $v$ u (neki) svoj svet

[irse/ir/salir + prep + [los cerros de Úbeda] 1 1. (alejarse de un tema) skrenuti s teme $v$, udaljiti se od teme $v$ 2. (evitar un tema) obilaziti kao kiša oko Kragujevca loc $v(\mathrm{col})$; okolišati $v \mathbf{3}$. (hablar o pensar demasiado) rasplinuti se $v$

[salir/salirse + prep + [los cerros de Úbeda]] (decir algo inesperadamente y fuera de propósito) lupiti / lupati / lupetati $v(\mathrm{col})$.

Estas equivalencias pueden considerarse prototípicas, identificadas a partir de la frecuencia de uso, pero no las únicas, como han mostrado también diferentes estudios de traducción de las unidades fraseológicas.

Se ha observado que la equivalencia contextual puede adoptar diferentes formas, y que a la construcción fraseológica de partida le puede corresponder tanto una construcción fraseológica en la lengua meta como una unidad léxica simple, aunque a veces, sobre todo en casos de modificaciones creativas, se prefiere el cambio de categoría, la descripción o incluso la creación de un neologismo.

Las modificaciones creativas de la construcción fraseológica en cuestión han confirmado que la variación es inherente a las unidades fraseológicas (Corpas Pastor 2011: 74), al igual que a otras unidades lingüísticas. Asimismo, se ha confirmado la excelente capacidad de la construcción fraseológica analizada de «adaptar su significado al contexto» (Torrent-Lenzen 2009: 544). 


\section{Fuentes}

CLAVE: VV.AA. CLAVE. Diccionario de uso del español actual. Disponible en http://clave.smdiccionarios.com

CORPES XXI: Real Academia Española: Banco de datos (CORPES XXI) [en línea]. Corpus del Español del Siglo XXI (CORPES). http://www.rae.es. 24.04.2021.

CREA: Real Academia Española: Banco de datos (CREA) [en línea]. Corpus de Referencia del Español Actual (CREA). http://www.rae.es. 24.04.2021.

DFDEA: Seco, M., Andrés, O. \& Ramos, G. (2004). Diccionario fraseológico documentado del español actual. Locuciones y modismos españoles. Madrid: Aguilar.

DFEM: Varela, F. \& Kubarth, H. (1996). Diccionario fraseológico del español moderno. Madrid: Gredos.

DLE: Real Academia Española. Diccionario de la lengua española (23a ed.). Disponible en www.rae.es

\section{Referencias bibliográficas}

Corpas Pastor, G. (2001). La creatividad fraseológica: efectos semántico-pragmáticos y estrategias de traducción. Paremia, (10), 67-78.

Corpas Pastor, G. (2003a). La traducción de la fraseología: técnicas y estrategias. En: G. Corpas Pastor (ed.), Diez años de investigaciones en fraseología: análisis sintáctico-semánticos, contrastivos y traductológicos (págs. 213-223). Frankfurt am Main: Vervuert Verlagsgesellschaft.

Corpas Pastor, G. (2003b): Fraseología y traducción. En: G. Corpas Pastor (ed.), Diez años de investigaciones en fraseología: análisis sintáctico-semánticos, contrastivos y traductológicos (págs. 245-273). Frankfurt am Main: Vervuert Verlagsgesellschaft.

Dobrovol'skiï, D. (2005). Sobre la equivalencia translingüística de los fraseologismos. En: J.d.D. Luque Duran \& A. Pamies Bertrán (eds.), La creatividad en el lenguaje: colocaciones idiomáticas y fraseología (págs. 359-379). Granada: Método.

Jakobson, R. (1959). On Linguistic Aspects of Translation. In: R. Brower (ed.), On Translation (págs. 232-239), Cambridge, Massachusetts: Harvard University Press. 
Mellado Blanco, C. (2015). Parámetros específicos de equivalencia en las unidades fraseológicas (con ejemplos del español y el alemán). Revista de Filología, (33), $153-174$.

Mellado Blanco, C. (2020). La desautomatización desde el prisma de la gramática de construcciones: un nuevo paradigma de la variabilidad fraseológica. Nasledje, (45), 17-34.

Morvay, K. (1996). Harri batez bi kolpe. Cuestiones de fraseología comparada. EUSKERA, (41/3), 719-767.

Pejovic, A. (2013). O ekvivalenciji i lažnim prijateljima u španskoj i srpskoj frazeologiji. Srpski jezik, (18), 401-414.

Pejovic, A. (2021). (Por) los cerros de Úbeda en el corpus del español: enfoque construccionista. Ponencia plenaria presentada en el LV Congreso de la AEPE.

Disponible en https://www.youtube.com/watch?v=CfGw $0 \quad \times 2 j 8$

Penadés Martínez, I. (2021). Las construcciones fraseológicas desde la perspectiva lexicográfica. $\quad$ LinRed, DIII. Disponible en http://www.linred.es/articulos pdf/LR-articulo-17072021.pdf

Schellheimer, S. (2016). La función evocadora de la fraseología en la oralidad ficcional y su traducción. Berlin: Frank \& Timme.

Sevilla Muñoz, M. (2015). Condicionantes textuales en la traducción fraseológica y paremiológica. Paremia, (24), 95-107.

Szerszunowicz, J. (2016). Frasemas cuasi-equivalentes en la fraseología teórica, la fraseografía y la traductología. Language Design, (16), 151-177.

Szyndler, A. (2014). Sobre la pseudo-equivalencia fraseológica desde una perspectiva cognitivista. Anuario de Estudios Filológicos, XXXVII, 251-267.

Timofeeva, L. (2012). Sobre la traducción fraseológica. ELUA, (26), 405-432.

Torrent-Lenzen, A. (2009). Internet, reto y revolución: las definiciones de las locuciones en los diccionarios y su verdadero significado fraseológico. En: P. Cantos Gómez \& A. Sánchez Pérez (eds.), Panorama de investigaciones basadas en corpus (págs. 534-547). Murcia: Universidad de Murcia.

Torrent-Lenzen, A. (2012). Los conceptos relativos a la equivalencia traductora en el campo de la fraseología: revisión crítica y propuestas. En: H. van Lawick \& B. Jurku (eds.), Übersetzen als Performanz. Translation und Translationswissenschaft in performativem Licht (págs. 273-286), Wien / Berlín: Lit Verlag. 
Trivic. A. (2013). Correspondencia recíproca en la fraseología. Análisis contrastivo español-serbio. Colindancias, (4), 69-80.

Zholobova, A. (2005). Acerca de la fraseología internacional: los europeísmos culturales. Interlingüística, (16/2), 1191-1197.

\section{Summary}

\section{THE PHRASEOLOGICAL CONSTRUCTION [Vmov + prep + [los cerros de Úbeda]] AND ITS POSSIBLE CONTEXTUAL EQUIVALENTS IN SERBIAN}

The main aims of this paper are the presentation of the Spanish phraseological construction [Vmov + prep + [los cerros de Úbeda]] and the nuances of its meaning in Spanish together with the identification of its equivalents in Serbian. For the purposes of our analysis, we use the theoretical framework developed by the Construction Grammar. The data has been obtained from two relevant corpora of the contemporary Spanish language, CREA y CORPES XXI, both developed by the Spanish Royal Academy and available online.

Our goal being the identification of equivalents of the Spanish phraseological construction in Serbian, first we address this key concept in the introduction. In effect, one of the main problems in Phraseology, both from a theoretical and practical point of view, is that of equivalence. Apart from Phraseology, the problem of equivalence has also been analysed from the perspective of Translation Studies. Thus many scholars in both disciplines mentioned above have proven through research that lexicographical equivalence is not the same as the (con)textual equivalence (Torrent-Lenzen, 2009, 2012; Timofeeva 2012; Mellado Blanco, 2015; Sevilla Muñoz, 2015). Moreover, Mellado Blanco (2015: 155) has found a key difference between lexicographical and textual equivalence. This difference lies in the fact that lexicographical equivalence does not pretend to reach or include all the possible translations of one particular phraseological unit from L1 to $L 2$. Such findings lead to the inevitable conclusion that a definition of a phraseological unit offered by either a monolingual or a bilingual dictionary will not necessarily always represent an appropriate solution for one specific translation.

The complexity of the phraseological meaning is the reason that lies beneath the fact that is it by no means an easy task to detect its equivalents, especially in a different language. As observed by Torrent-Lenzen (2009: 544), phrases possess the ability of adapting their meaning to context and by doing this, following Sevilla Muñoz (2015: 96), they acquire a meaning that is unique. Through analysis of the use of the Spanish phraseological construction in context, we observe that it possesses a wider range of meanings than the ones usually presented in monolingual dictionaries. More precisely, we have found that the Spanish phraseological construction [Vmov + prep + [los cerros de Úbeda]] has five meanings. Following one of the main aims of our paper, we then identify their contextual equivalents in Serbian. For the presentation of the senses in Spanish and their equivalents in Serbian, we use the lexicographical model defined by Penadés Martínez (2021). Although we use the model previously mentioned, it needs to be specified that we have adapted it for the purposes of our analysis.

In analysing the Spanish phraseological construction [Vmov + prep + [los cerros de Úbeda]] we further find in the examples from the corpus representing real use that it presents modifications. On some occasions, these modifications can affect the structure of the appropriate equivalent in Serbian.

In line with another key concept within the field of Phraseology, that of cognitive entrenchment, one of the conclusions of our analysis is that the modifications of the phraseological construction and its variation do not impede the communicative process and, therefore, do not stand in the way of it being understood.

Key words: equivalence, Phraseology, corpus, translation, Construction Grammar, Spanish, Serbian. 